reduction of $\Delta$ from the $3,750 \mathrm{~cm} .^{-1}$ provisionally estimated by $\mathrm{Orgel}^{10}$ and the $3,500 \mathrm{~cm}^{-1}$ used by Ballhausen and Jorgensen ${ }^{11}$. The molecular extinction coefficients given by Orgel $^{10}$ are also too large by a factor of five.

We have also been unable to confirm the spectrum claimed ${ }^{12}$ for $\mathrm{NiCl}_{4}{ }^{2-}$, which was based on bands found in the spectrum of a complex melt; we consider that the melt spectrum belongs to some other (perhaps essentially square or tetrahedral) species of nickel halide, since it closely resembles a spectrum we obtained when anhydrous nickel chloride was dissolved in dimethylformamide, dimethylacetamide or dimethyl sulphoxide. The spectrum of the $\mathrm{NiCl}_{4}{ }^{2-}$ species in a 'Nujol' mull has one broad band with a maximum between 7,500 and $7,600 \mathrm{~cm}^{-1}$, and on simple crystal field theory it is impossible to account for a band at that energy unless a value of $\Delta$ larger than $4,000 \mathrm{~cm}^{-1}$ is used. From other compounds this seems too large, and it can be reduced to about $3,650 \mathrm{~cm} .^{-1}$ if the complete treatment including spinorbit interaction ${ }^{7}$ is used for the calculations. Even this value seems large, and it may be that there are relatively large non-cubic components of the perturbing field in this molecule because of departures from a perfectly tetrahedral structure.

The values of $\Delta$ for cobrlt and manganese are close to one-third the value of $\Delta$ (octahedral) for the hexahydrates. If, as has been suggested ${ }^{9}$, the $D q$ for $\mathrm{Cl}^{-}$is only 75 per cent that of water, then the theoretical ratio of $4 / 9$ between the octahedral and tetrahedral $\Delta$ values would be well supported by experiment.

A complete report of this work is now being prepared for publication.

\section{S. BUFFAGNi*}

T. M. DUNN

William Ramsay and Ralph Forster Laboratories, University College London, Gower Street,

London, W.C.1.

* Present address: Istituto di Chimica Generale, Università di Modena, Italy.

${ }^{\prime}$ Gill and Nyholm, J. Chem. Soc., 3997 (1959).

2 Gill, Nyholm and Pauling, Nature, 182, 168 (1958).

${ }^{3}$ Libus, Ugriewska and Minc, Ruczniki Chemii, 34, 29 (1960).

- Katzin and Geberg, J. Amer. Chem. Soc., 72, 5464 (1950).

'Cotton and Francis, J. Amer. Chem. Soc., 82, 2986 (1960).

- Jorgensen and Ballhausen, Acta Chem. Scand., 9, 397 (1955).

'Liehr and Ballhausen, Annals of Physics, 6, 134 (1959).

${ }^{8}$ Cotton and Holm, J. Chem. Phys, 32, 1168 (1960).

9 Jorgensen, Proc. Tenth Solvay Gonference, 355 (1956).

${ }^{10}$ Orgel, J. Chem. Phys., 23, 1004 (1955).

${ }^{11}$ Balthausen and Jorgensen, Acta Chem. Scand., 9, 397 (1955),

${ }^{12}$ Jorgensen, Mol. Phys., 1, 410 (1958).

${ }^{13}$ Jorgensen, Acta Chem. Scand., 11, 53 (1957).

"Dunn, Proc. of the Symp, on Molecular Spectroscopy, Bologna, September 1959 (Pergamon Press, in the press).

${ }_{15}$ Dreisch and Trommer, $Z$. physik. Chem., $\mathrm{B} 37,37$ (1937).

\section{BIOCHEMISTRY}

\section{A Possible Biochemical Mechanism for Memory}

IN a recent paper, Leo Szilard has shown that a system consisting of an enzyme, an enzyme-forming apparatus and a repressor may be able to exist in two stable states, provided the combining energies between the various components of this system lie within certrin ranges of magnitude. The two stable states which can exist contain either high or low concentrations of the enzyme. If the concentration of the enzyme or the repressor is changed by external manipulation, the system returns to one or the other of the stable states. Such a system was termed 'para-constitutive' by Szilard, and its possible biological significance in differentiation and antibody formation ${ }^{2}$ was suggested by him.

I should like to direct attention to another possibility, namely, that a para-constitutive system, the components of which are at this time beyond conjecture, may constitute the unit of memory in the brain. A change from one to the other of the two stable states would correspond precisely to the binary memory components used in computors. Since a human brain cell may contain as many as $10^{6}$ genes, as estimated by Szilard for other human cells, a very large number of binary units per brain cell seem possible. It appears conceivable that one might obtain experimental information on the existence of such systems by the careful analysis of brain constituents as a function of the number of memory impulses to wrich the brain was exposed. A brain exposed to a large number of sensory stimuli would bo expected to have different concentrations of the components of the postulated system from a brain carefully shielded from such stimuli.

I should like to suggest that 'para-constitutive' systems may have numerous other biological applications, and that the recognition of their existence appears to be one of the most important biological hypotheses made in recent times.

H. S. ANKER

Department of Biochemistry,

University of Chicago.

${ }^{1}$ Szilard, L., Proc. U.S. Nat. Acad. sci., 46, 27i (1960).

"Szilard, L., Proc. U.S. Nat. Aerd. Sci., 46, 293 (1960).

\section{Heterogeneity of Fatty Acids from Royal Jelly}

THE presence of hydroxy fatty acids as the major component of the lipid portion of royal jelly was indicated many years ago by Townsend and Lucas ${ }^{1}$ and was confirmed recently by Butenandt and Rembold ${ }^{2}$. The latter authors characterized the major component as 10-hydroxy-2-decenoic acid. A trans configuration has been assigned to the double bond ${ }^{3,4}$. This unusual fatty acid has been found also in the food of worker bee larvæ2 and in the mandibular glands of worker bees ${ }^{3,5}$, but appears to be absent from pollen and other bee food. Unusual biological activity has been aseribed to the substance by Blum et al. ${ }^{6}$ and by Townsend et al. ${ }^{7}$.

Several chromatographic methods have been employed in our Laboratories for a careful examination of the chloroform-soluble portion of lyophilized royal jelly. The results have revealed that the lipid portion is a mixture which is resolved with difficulty and is somewhat more complex than has been previously supposed. A large fraction of the royal jelly lipids consists of free fatty acids. Chromatography of the chloroform extract on a silicic acid adsorption column separated the major acidic components into three fractions. The first of these consists of decandioic acids, the second consists of several monohydroxy monocarboxylic acids, and the third consists principally of dihydroxy monocarboxylic acids. Each fraction was separated further by silicic acid partition chromatography, paper chromatography, and chrom- 